Apidologie, 1977, 8 (1) 1-18.

\title{
TRANSFER DER SPERMATOZOEN IN DIE SPERMATHEKA DER BIENENKÖNIGIN
}

\author{
Le transfert des spermatozö̈des dans la spermathèque \\ de la reine d'abeille \\ B. GESSNER und F. RUTTNER \\ Institut für die Bienenkunde (Polytechnische Gesellschaft) \\ der Universität Frankfurt Oberursel B.R.D.
}

\section{SUMMARY}

TRANSFER OF THE SPERMATOZOA

INTO THE SPERMATHECA OF THE HONEY BEE QUEEN

The active migration and the passive transport of the spermatozoa into the spermatheca of the queen bee are studied by a number of experiments.

Warm Ringer solution poured on the spermathecal duct in the dissected abdomen releases contractions of its muscles. Balls of polyethylen of $7 \mu \mathrm{m}$ diameter injected as suspension into the queens oviducts are transported into the spermatheca, though in a small proportion only (in the range of a factor $3.10^{-2}$ compared to spermatozoa of the same volume injected).

The speed of migration of spermatozoa is highest in ducts of less than $35 \mu \mathrm{m}$ diameter.

For active migration into the spermatheca the spermatozoa need the activation by the liquid within the duct.

Except into the spermatheca, the spermatozoa migrate also into the peripheric ducts of the spermathecal gland.

A diagram is designed to illustrate the complex cooperation of all the factors described in the process of filling the spermatheca.

\section{ZUSAMMENFASSUNG}

In einigen Versuchen wird die aktive Wanderung und der passive Transport der Spermatozoen in die Samenblase untersucht. 
Durch Einwirkung warmer Ringerlösung auf den freipräparierten Samenblasengang lassen sich Kontraktionen seiner Muskulatur auslösen. Polyäthylenkügelchen von $7 \mathrm{\mu m}$ Durchmesser in Form einer Suspension in die Ovidukte injiziert, können in die Spermatheka gelangen, allerdings mit dem Faktor $3.10^{-2}$ weniger als Samenfäden nach Injektion eines gleichen Volumens.

Die Wandergeschwindigkeit der Spermatozoen ist in Kanälen mit einem Durchmesser von $35 \mu \mathrm{m}$ und darunter am höchsten.

Voraussetzung für die aktive Einwanderung der Spermatozoen in die Spermatheka ist deren Aktivierung durch die Flüssigkeit im Ductus spermaticus. Die Spermien wandern nicht nur in die Samenblase sondern auch in die peripheren Gänge der Spermathekaldrüse.

Es wird versucht, das komplexe Zusammenspiel dieser Faktoren durch ein Blockdiagramm anschaulich zu machen.

\section{EINLEITUNG}

Nach der Paarung der Bienenkönigin werden die Spermatozoen bis zu 24. Stunden in den beiden lateralen Ovidukten gelagert. Die Menge der während dieser Zeit in die Spermatheka der Königin transferierten Spermatozoen hängt von dem jeweiligen Füllungsgrad der Ovidukte nach der Paarung ab. Auf Grund einer ausführlichen Diskussion über diesen Vorgang (RuTTNER, 1956) und von experimentellen Untersuchungen zu einigen wesentlichen Punkten des Spermatransfers (Ruttner und Koeniger, 1971) lassen sich die derzeitigen Kenntnisse und Hypothesen folgendermaßen darstellen (Blockdiagramm Abb. 1).

Infolge der Wanddehnung während der Füllung der Ovidukte (A) mit Sperma werden - möglicherweise durch Erregung motorischer Zentren des Abdomens (E) - Kontraktionen der Abdominalmuskulatur ausgelöst (B). Hierdurch wird das Sperma kaudal durch die Vagina und vorbei an der Öffnung des Spermathekalganges nach außen gepreßt. Nach Blockierung der Kontraktionsmuskulatur kommt es weder zur Entleerung der Ovidukte noch zur Füllung der Spermatheka (Ruttner und Koeniger, 1971). Eine Voraussetzung für das Eindringen in den Spermathekalgang und für die Passage ist eine Orientierungsreaktion der Spermatozoen (C). Inaktivierte Spermatozoen gelangen nicht in die Spermatheka (D). Nach Ifantidis (1972) sind Bienenspermatozoen zu einer aktiven Lokomition befähigt. Dennoch ist nicht bekannt, ob dies bei der Passage durch den D. spermaticus eine wesentliche Rolle spielt (F). Kontraktionen der Spermathekalpumpe (I) und gerichtete aktive Bewegungen der Spermatozoen (F) durch Chemotaxis (H) bzw. Rheotaxis (N) können den Spermatozoentransfer wesentlich beeinflussen.

Nach den bisherigen Untersuchungen kann nicht angegeben werden, ob die alleinige Kontraktion der Oviduktmuskulatur (G) und der Spermathekalpumpenmuskulatur (K) zur Füllung der Spermatheka ausreicht. 
Als Ergebnis der Überlegungen zu diesem Schema sollen folgende Einzelprobleme des Spermatozoentransfers näher untersucht werden :

1. Funktionsbeschreibung der Muskulatur des Spermathekalganges (“ Samenpumpe )).

2. Transport unbelebter Körper entsprechender Größe aus den Ovidukten in die Spermatheka.

3. Modellversuch zum Nachweis der Lokomotion von Spermatozoen in einem Gang geringen Durchmessers, entsprechend dem Lumen des Samenblasenganges.

4. Bedeutung eines möglichen innerspermathekalen Druckes für den Spermatransfer.

5. Verhalten der Spermatozoen in den Geschlechtswegen der Königin.

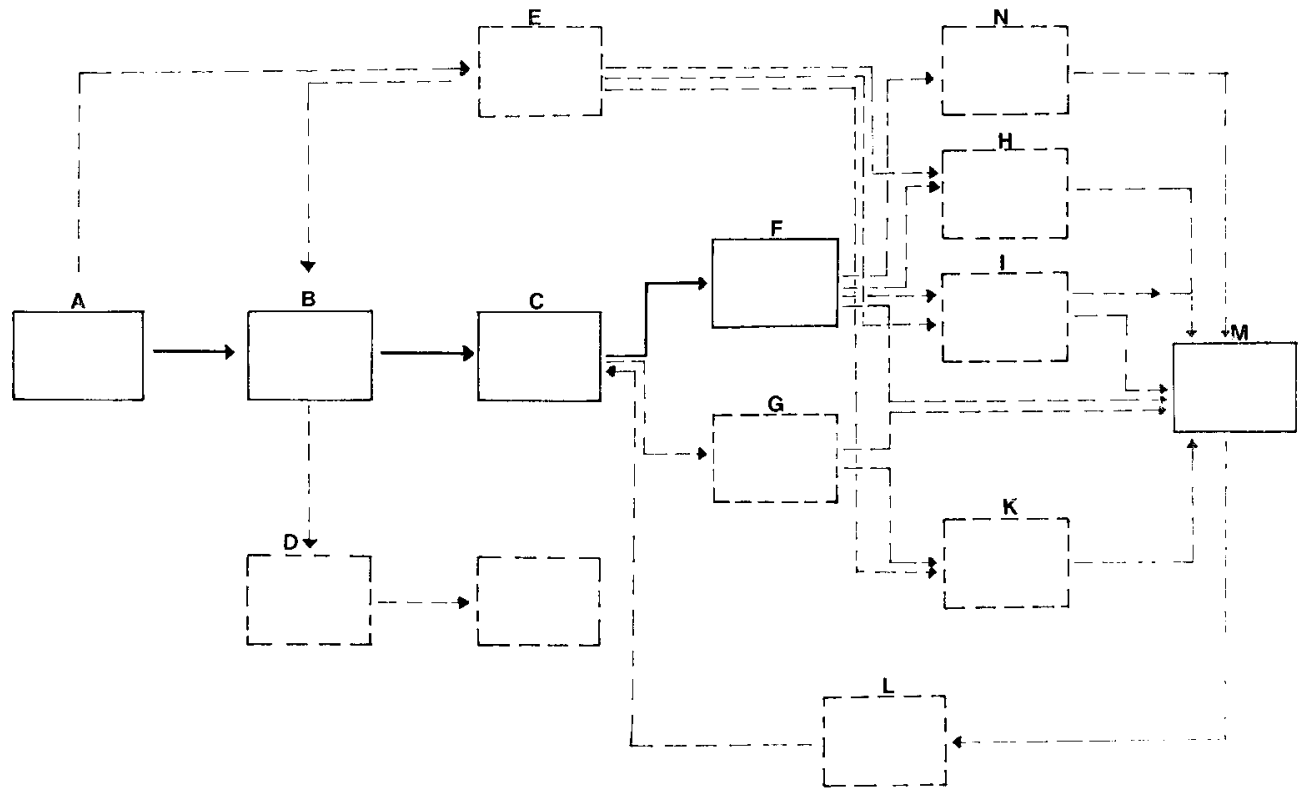

Aв8. 1. - Schematische Ubersicht zum Vorgang des Transfers der Spermatozoen aus den Ovidukten in die Spermatheka.

Ausgezogene Linien : Experimentell abgesicherte Vorgänge. Gestrichelte Linien : Denkbare, aber bisher rein hypothetische Wege. U̇brige Erklärungen im Text.

FIG. 1. - Vue gênérale schématique du mécanisme de transfert des spermatozoüdes des oviductes dans la spermathèque.

Lignes continues : mécanismes vérifiés expérimentalement.

Tirets : trajets possibles, mais jusqu'à présent purement hypothétiques.

Explications dans le résumé. 


\section{1. - Funktion der « Samenpumpe »}

\subsection{Methode}

Bei einer inseminierten Königin, die einige Stunden nach der Inseminierung in Ringerlösung (Zimmertemperatur) aufpräpariert worden war, konnten eindeutig Kontraktionen der Muskulatur des Ductus spermaticus festgestellt werden. Die anfängliche Frequenz betrug etwa 200/min. Wie G. Konniger (pers. Mitteilung) beobachtete, ließen sich solche Kontraktionen auch bei unbesamten Königinnen auslösen, wenn man die freigelegten Geschlechtsorgane mit einer erwärmten Ringerlösung $\left(35-40^{\circ} \mathrm{C}\right)$ übergießt. Mit dieser Methode kann die Reaktion nach Belieben reproduziert und ihr Verlauf unter einem Stereomikroskop beobachtet werden. Zur Analyse wurde der Bewegungsablauf mit einem Videorekorder registriert und später jede einzelne Phase von einem Monitor abgezeichnet.

\subsection{Ergebnisse}

Die Kontraktionen des Samenblasenganges halten einige Minuten an, worauf sowohl Frequenz wie Amplitude abnehmen. Ob ein peristaltischer Bewegungsverlauf vorliegt, kann wegen der hohen Frequenz und des geringen Bildauflösungsvermögens der Kamera nicht sicher gesagt werden. Die stärkste Kontraktion scheint unmittelbar unterhalb dem S-förmigen Knick der Samenpumpe zu erfolgen.

Der Kontraktionszustand ist auf Abb. 2 schematisch dargestellt. Die skizzierte Verengung der Ganglichtung konnte am lebenden Objekt nicht beobachtet werden, da die Wände des Ductus nicht durchsichtig sind; bei der starken Kontraktion der Ductuswand ist sie aber zwingend anzunehmen.

\section{2. - Transport unbelebter Körper in die Spermatheka}

\subsection{Methode}

Die Spermatozoen der Honigbiene haben einen Durchmesser von $0,5 \mu \mathrm{m}$ und eine Länge von $300 \mu \mathrm{m}$ (Rothschild, 1955). Der Durchmesser des Kopfes ist dabei nicht wesentlich größer als der der Geißel. Wie schon oben gesagt, gelangen inaktivierte Spermatozoen nicht in die Spermatheka.

Um die hier gestellte Frage zu beantworten, wurden daher als inaktive Objekte Polyäthylenkügelchen von $7 \mu \mathrm{m}$ Durchmesser (Serva, Heidelberg) gewählt. Die Kügelchen sind in Latexmilch suspendiert. Um die Viskosität der Suspension zu erhöhen, wurde sie zu gleichen Teilen mit einer $10 \%$ igen 
Gelatinelösung gemischt. Mit dieser Latex-Gelatine-Suspension wurden junge unbegattete Königinnen nach der üblichen Methode mittels einer Kanüle inseminiert. Pro Königin wurde $6 \mu$ l Suspension verwendet, was etwa einer Anzahl von 4,5 Mill. Kügelchen entspricht.
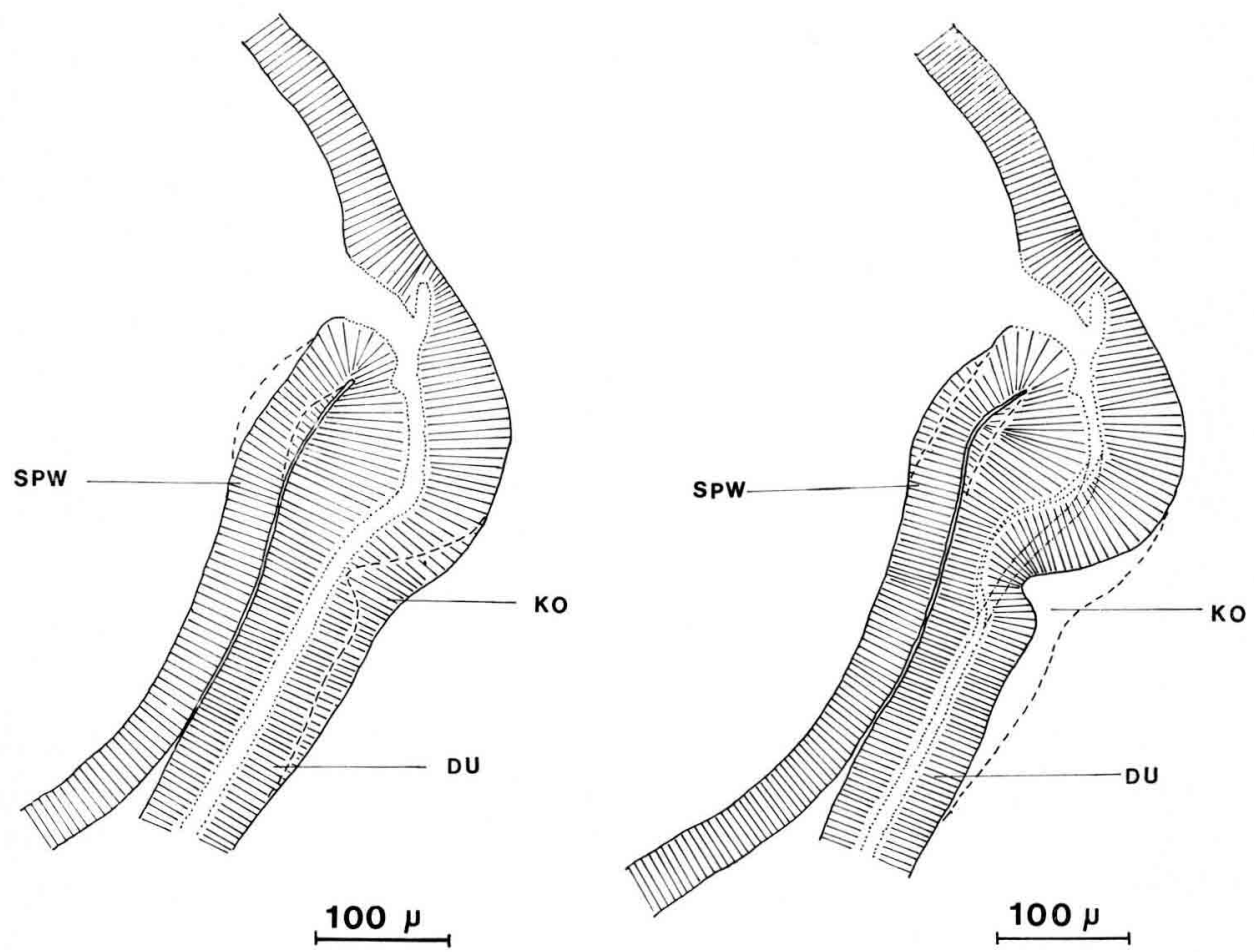

Авв. 2.- Schematischer Schnitt durch Ductus spermaticus (DU) und Spermathekalwand (SPW). Links im Ruhezustand, rechts kontrahiert. KO Stelle maximaler Kontraktion.

FIg. 2. - Coupe schématique à travers le ductus spermaticus (DU) et la paroi de la spermathèque (SPW). A gauche : au repos; à droite : en contraction; KO : lieu de contraction maximum

\subsection{Ergebnisse}

7 Königinnen, besamt mit $6 \mu$ l der Suspension, wurden 48 Stunden nach dem Eingriff präpariert. Zu diesem Zeitpunkt waren die Ovidukte in allen Fällen frei von der injizierten Suspension. Die Auszählung der Kügelchen erfolgte in einer Zählkammer nach Fuchs-Rosenthal (Tab. 1).

Es war also eine gewisse Anzahl Polyäthylenkügelchen (im Mittel 1140 \pm 485 ) aus den Ovidukten durch den engen, etwa $1 \mathrm{~mm}$ langen Kanal in die Spermatheka gelangt. Bei der Beurteilung dieser Zahl ist zu berücksichtigen, daß nach Mackensen und anderen (vgl. Ruttner 1975) nach Besamung mit 
$6 \mu l$ Sperma im Mittel 3,3-4,9 Mill. Spermatozoen in der Spermatheka gefunden wurden, das sind etwa 5-7,5\% der injizierten Menge.

TAB. 1. - Anzahl der Polyäthylenkugeln in der Spermatheka von Königinnen, die mit $6 \mu$ l einer Suspension dieser Kugeln besamt worden waren.

TABL. 1. - Nombre de billes de polyéthylène dans la spermathèque des reines que l'on a inséminées avec $6 \mu l$ d'une suspension de ces billes.

\begin{tabular}{|c|c|}
\hline $\begin{array}{c}\text { Königin No } \\
\text { Reine } \mathrm{N}^{\circ}\end{array}$ & $\begin{array}{c}\text { Anzahl der Polyäthylenkugeln } \\
\text { in der Spermatheka } \\
\text { Nombre de billes de polyéthylène } \\
\text { dans la spermathèque }\end{array}$ \\
\hline $\begin{array}{l}1 \\
2 \\
3 \\
4 \\
5 \\
6 \\
7\end{array}$ & $\begin{array}{cc}1000 \\
1500 \\
1500 \\
625 \\
1000 \\
875 \\
1500 \\
\overline{\mathbf{x}}=1140 \\
\mathrm{~s}= \pm 385\end{array}$ \\
\hline
\end{tabular}

Bei gleicher Effizienz des Transfers der Kügelchen wären 342.000 von ihnen in der Spermatheka zu erwarten gewesen - etwa 300 mal soviel als tatsächlich gezählt wurden. Allerdings sind sowohl Form wie Durchmesser der Kügelchen ganz anders als bei den Spermatozoen.

\section{3. - Lokomotion der Spermatozoen}

\subsection{Methodik}

Bei den bisherigen Versuchen zu diesen Fragen war es nicht gelungen, Versuchsbedingungen zu schaffen, die den natürlichen Verhältnissen entsprechen. Ifantidis (1970) untersuchte im hängenden Tropfen, wobei sich die Spermatozoen nach allen Seiten frei ausbreiten konnten. G. Koeniger (1970) verwendet beidseitig verschlossene Glaskapillaren von etwa $200 \mu \mathrm{m}$ Durchmesser - also dem 10-fachen Durchmesser des D. spermaticus.

Wir bedienten uns einer anderen Methode : Die Vertiefung eines Hohlschliff-Objektträgers wird mit Paraffinöl ausgefüllt. Auf der einen Seite des Hohlschliffs wird aus einer Kapillare auf dem Boden des Objektträgers (also unter Luftabschluß) Sperma zu einem dünnen Strang ausgezogen (Abb. 3, S). Senkrecht dazu füllt man mittels einer zweiten Kapillare, ebenfalls unter Öl 
auf dem Boden des Hohlschliffs, einen weiteren Kanal (T) mit einer Testflüssigkeit (physiol. Kochsalzlösung oder andere Lösungen). Die Breite des Testkanals kann durch die Wahl von Kapillaren verschiedenen Durchmessers, bzw. der Auszugsgeschwindigkeit nach Belieben verändert werden.

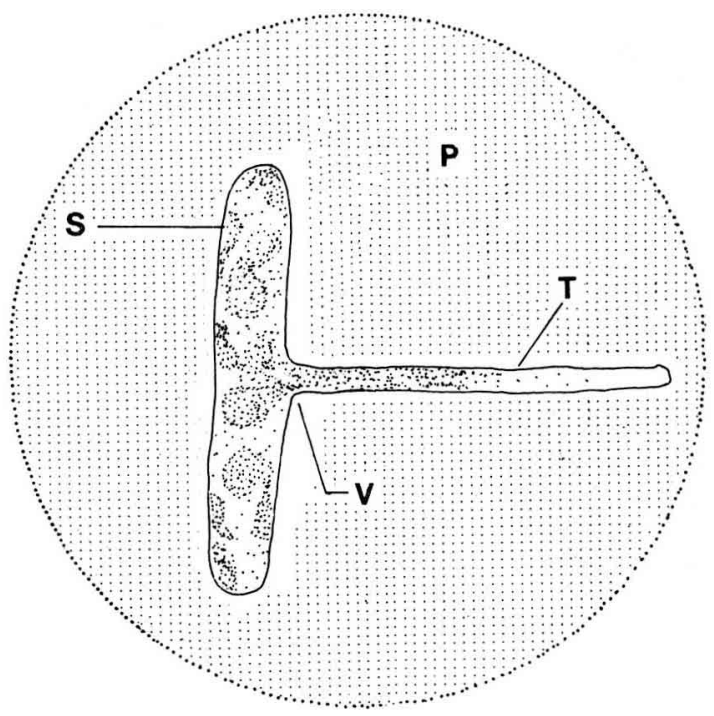

Авв. 3. - Versuchsaufbau zur Messung der Spermatozoengeschxindigheit in verschiedenen Lösungen und in Kanälen verschiedener Breite.

P Paraffin, S Kanal mit Sperma, T Kanal mit Testsubstanz, V Verbindungsstelle.

FIG. 3. - Montage expérimental pour la mesure de la vitesse des spermatozoüdes dans diverses solutions et dans des canaux de divers diamètres.

$\mathbf{P}$ : paraffine; $\mathrm{S}$ : canal contenant le sperme; $\mathrm{T}$ : canal contenant la substance à tester; $\mathrm{V}:$ jonction

Mit der Spitze einer Glaskapillare wird die Verbindung (V) zwischen den beiden Kanälen hergestellt. Die Wanderungsgeschwindigkeit der Spermatozoen bis an das Ende des Testkanals läßt sich jetzt leicht bestimmen.

\subsection{Ergebnisse}

Als Testflüssigkeiten zur Bestimmung der Fortbewegungsgeschwindigkeit der Spermatozoen dienten Spermathekalflüssigkeit, Hämolymphe von Arbeitsbienen und physiologische Kochsalzlösung. In der Ringerlösung verminderte sich die Motilität der Spermien nach einer äußerst lebhaften Anfangsphase innerhalb weniger Minuten. Sie krümmten sich z.T. unter krampfartigen Zuckungen zu einem Ring und blieben von nun an bewegungslos.

Auch in Hämolymphe konnten wir eine Inaktivierung der Spermatozoen nach fünf bis zehn Minuten feststellen. Da die Spermien innerhalb dieser Zeit 
in den meisten Fällen das Ende des Kanals erreicht hatten, ließ sich hier im Gegensatz zur physiologischen Kochsalzlösung aber ihre W anderungsgeschwindigkeit errechnen. Der Zeitpunkt völliger Immobilität war weitaus weniger exakt zu bestimmen als in Ringerlösung, da ihre Bewegungen bis zum bewegungslosen Zustand sehr allmählich abnahmen.

In der Spermathekalflüssigkeit zeigten die Spermatozoen eine etwas geringere Motilität als in den beiden anderen Testsubstanzen. Auch hier stellten sie die Bewegungen nach Erreichen des Kanalendes und einem Auffüllen des Ganges zu einem dicht gepackten Bündel ein, doch es war selbst nach Stunden durch Erweitern des Kanals oder durch Hinzufügen neuer Testflüssigkeit möglich, ihre alte Motilität wieder herzustellen.

Da durch die gewählte Methode nur ein zweidimensionales Sehen in der Ebene möglich war, konnten wir anstelle der von Ifantidis (1972) im hängenden Tropfen beschriebenen schraubenförmigen Fortbewegung nur eine wellenförmige Bewegung beobachten. Sofort nach der Verbindung des Kanals mit dem Spermastrang strebten einzelne Spermatozoen zum entfernten Kanalende, denen etwa in halber Länge ein dichter Pulk lebhafter Spermien folgte.

Zur Bestimmung der Geschwindigkeit wurden nur die ersten, also nur die schnellsten Spermatozoen gewertet. Während ihrer sinusförmigen Wellenbewegung stießen sie immer wieder gegen den Rand des Kanals, um sich dann zur gegenüberliegenden $W$ andseite zu drehen.

Die Fortbewegungsgeschwindigkeit war stark abhängig von der Amplitude dieser Sinusbewegung. Nach unseren Beobachtungen erreichten die Spermatozoen dann eine maximale Geschwindigkeit, wenn die Gangbreite um etwa 1/3 kleiner als die Dimension war, die eine Kehrtwendung der Spermien gestattet hätte. Diese optimale Gangbreite liegt im Bereich des mittleren Durchmessers des Samenganges (25 $\mu \mathrm{m}$; Rutrner 1956).

Allerdings waren Werte für die Geschwindigkeit in Kanalbreiten unter $30 \mu \mathbf{m}$ kaum zu gewinnen, da in engen Kanälen die Ränder durch die Kohäsionswirkung der jeweiligen Flüssigkeit sehr schnell zusammenflossen und damit der Gang unterbrochen wurde.

Andererseits war eine deutliche Verringerung der Spermatozoengeschwindigkeit zu erkennen, wenn der Kanal experimentell auf 100-200 $\mu \mathrm{m}$ erweitert wurde. Wir konnten beobachten, daß sich die vordersten Spermatozoen nach Erreichen der Erweiterung völlig umdrehten und damit dem nachfolgenden Pulk den Weg blockierten. Die Vorwärtsbewegung wurde plötzlich ungerichtet, und eine Fortbewegung kam nur dadurch zustande, daß die vordersten Spermien durch die nachfolgenden langsam weitergeschoben wurden.

Die im Pulk nachdrängenden Spermatozoen bewegten sich, durch ihre starke Packungsdichte veranlaßt, z.T. synchron, teilweise aber auch einzeln 
und strebten immer nach Stellen geringerer Spermatozoendichte. Befand sich eine solche manchmal tropfenförmige Erweiterung am Ende des Kanals, dann stellten die Spermien innerhalb weniger Minuten nach Erreichen einer kompakten Dichte ihre Bewegungen ein. Sie wurden aber sofort wieder aktiv, wenn mehr Raum gegeben wurde. Folgte nach einer Erweiterung erneut eine Verengung, so erhöhte sich sofort wieder die Geschwindigkeit, indem mehrere Spermatozoen dem Pulk in geringem Abstand vorausschwammen.

Die ermittelte Geschwindigkeit in Abhängigkeit zur Gangbreite wurde in Abb. 4 graphisch dargestellt. Worauf die niedrigere Spermatozoengeschwindigkeit in der Spermathekalflüßigkeit gegenüber der Hämolymphe zurückzuführen ist, konnte nicht geklärt werden.

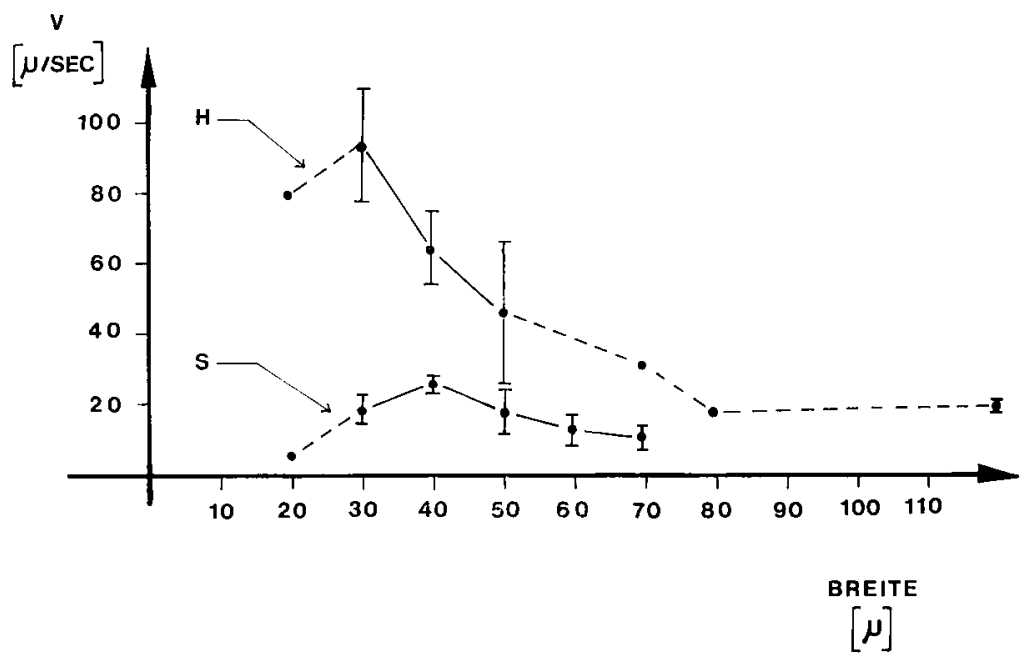

Aвв. 4. - Wanderungsgeschwindigkeit der Spermatozoen in Abhängigkeit von der Gangbreite (Abszisse). Die mit unterbrochenen Linien verbundenen Werte konnten wegen zu geringer Anzahl von Beobachtungen statistisch nicht abgesichert werden. H Wanderung in Haemolymphe, $S$ in Spermathekalflüssigkeit.

FIg. 4. - Vitesse de migration des spermatozö̈des en relation avec la largeur du passage (abscisse). Les valeurs reliées par des tirets n'ont pu être vérifiées statistiquement en raison du nombre trop restreint des observations. H : migration dans l'hémolymphe; $\mathrm{S}$ : dans le liquide spermathécal.

\section{4. - Punktion der Spermatheka}

\subsection{Methodik}

Nach einer leicht abgewandelten Methode von G. Koeniger (1970) wurden unbegattete Königinnen in einem Mikromanipulator fixiert und mit $\mathrm{CO}_{2}$ narkotisiert. Unter sterilen Bedingungen wurde in das 5 . Tergit ein Fenster geschnitten und die freigelegte Spermatheka vorsichtig mit einer feinen 
Glaskapillare punktiert, so daß Spermathekalflüssigkeit austreten konnte. Die Drüsen blieben intakt. Durch Zurückklappen des herausgeschnittenen Chitinstückes wurde die Wunde verschlossen. Die Behandlung der KontrollKöniginnen erfolgte in derselben Weise, nur mit dem Unterschied, daß das Anstechen der Spermatheka unterblieb.

Eine Stunde nach der Operation erfolgte die instrumentelle Besamung mit $4 \mu$ Sperma. 3-48 Stunden nach der Besamung wurden die Spermatozoen in der Spermatheka ausgezählt. Zur Untersuchung gelangten 7 Versuchs- und 7 Kontrollköniginnen.

Für den Versuch 4.22 wurde die Spermatheka anschließend an die Punktion so weit als möglich ausgesaugt.

\subsection{Ergebnisse}

\section{Punktion}

Zum Zeitpunkt der Präparation waren die Spermatheken in allen Fällen mit lebenden Spermatozoen gefüllt, die lebhafte Aktivität zeigten. Nur in der Gegend der Einstichstelle hatten sich ungeordnete Knäuel abgestorbener Spermien gebildet. Wie später noch genauer nachgewiesen werden konnte (s. unten), ist dies ein Effekt der Berührung mit Hämolymphe, die die Spermatozoen inaktiviert. Das Eindringen von Hämolymphe in die Spermatheka ist ein Beweis dafür, daß durch die Punktion eine offene Verbindung zwischen dem Innenraum der Spermatheka und dem Hämolymphraum hergestellt worden war.

Der Füllungsgrad der untersuchten Spermatheken ist in Abb. 5 dargestellt. Es zeigte sich, daß im Endzustand zwischen punktierten und nicht punktierten Samenblasen kein Unterschied bestand. Die Einwanderung schien bei den ersteren sogar rascher zu erfolgen, weil bei ihnen der maximale Füllungsgrad schon nach weniger als 12 Stunden erreicht war.

\section{Absaugen der Spermathekalflüssigkeit}

Bei einer zweiten Versuchsserie wurde zusätzlich zur Punktion die Spermathekalflüssigkeit abgesaugt. Das hatte zur Folge, daß die Samenblasenwand vollständig kollabierte. Eine Stunde nach dem Eingriff wurde mit $4 \mu$ Sperma besamt. Die Auszählung der in die Spermatheka gelangten Spermatozoen erfolgte 3-18 Stunden nach der Besamung (Abb. 6).

Bei der Präparation zeigte sich, daß die Spermatheka schon 4 Stunden nach der Operation ( $=3$ Stunden nach der Besamung) weitgehend ihre alte Form wieder angenommen hatte. Dies war nicht Folge einer Sekretion in die Blase, denn die Wiedererlangung der alten Form war auch dann zu beobachten, wenn in die $W$ and ein größeres, viereckiges Fenster geschnitten und anschlie- 
Bend die Blase zusammengepreßt worden war. Es verhält sich vielmehr so, daß die Spermatheka innen mit einer durchsichtigen, sehr festen Cuticula ausgekleidet ist, die wie ein Gummiball die Tendenz hat, nach jeder Deformierung wieder die alte Gestalt anzunehmen.

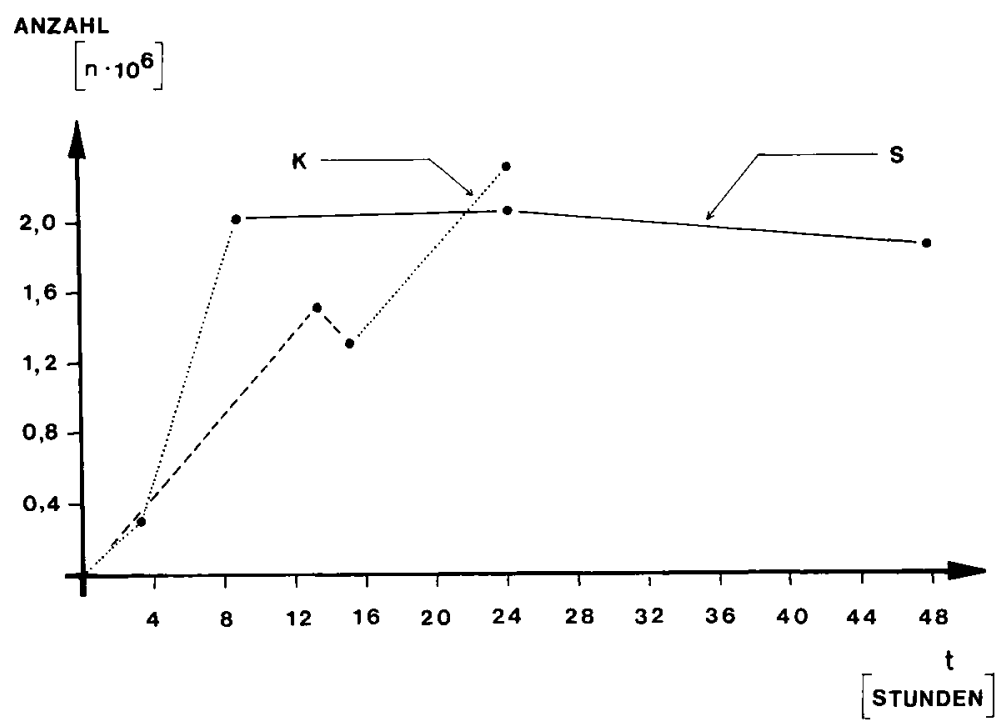

Aвв. 5. - Einwanderung der Spermatozoen (in Millionen) in punktierte Spermatheken (S). K Kontrolle mit intakten Spermatheken. Durch Punkte verbundene Werte : Statistisch nicht gesichert.

FIg. 5. - Migration des spermatozoïdes (en millions) dans la spermathèque après ponction (S) $\mathrm{K}$ : contrôle avec une spermathèque intacte.

Valeurs reliées par des pointillés : valeurs non vérifiées statistiquement.

Die schon wenige Stunden nach dem Aussaugen in der Spermatheka wieder vorhandene Flüssigkeit dürfte zum größten Teil Hämolymphe gewesen sein. Wie nach der Punktion waren auch hier die Spermatozoen in der Nähe der Einstichstelle verklumpt, jedoch waren auch alle übrigen unbeweglich.

Wie die Auszählung ergeben hat, war schon nach 3 Stunden ein Plateau von etwa 10.000 eingewanderten Spermien erreicht, das sich im weiteren Verlauf nicht mehr änderte (Abb. 6). Wenn die festgestellten Zahlen also um mehr als zwei Zehnerpotenzen unter denen liegen, die nach einfacher Punktion gefunden wurden (Abb. 5), so ist das im wesentlichen darauf zurückzuführen, daß der Einwanderungsproze $\beta$ zwar in Gang kam, aber sehr rasch wieder abgestoppt wurde. 


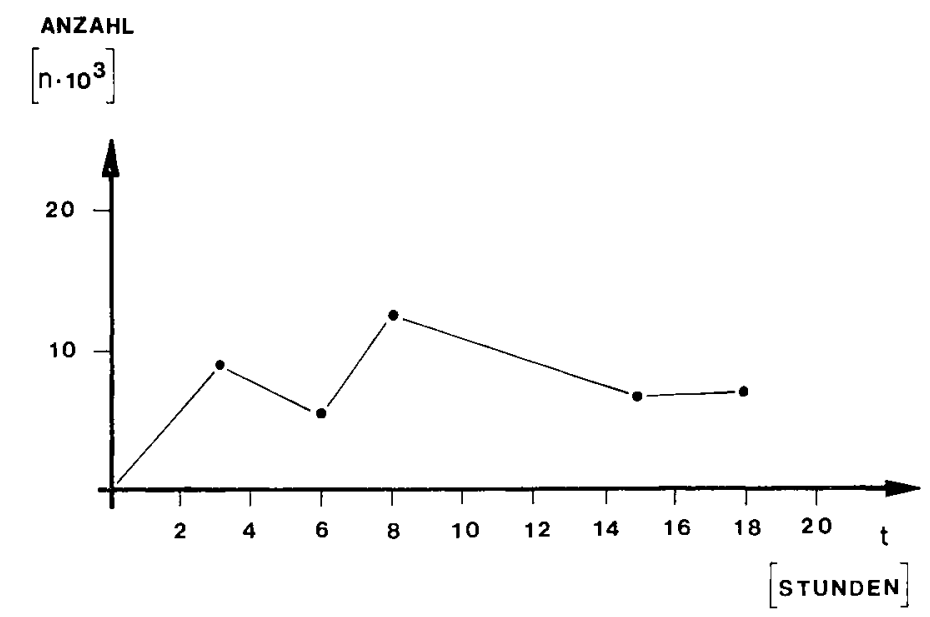

Aвв. 6. - Einwanderung der Spermatozoen (Angabe in Tausend) in Spermathelen, die nach der Punktion ausgesaugt worden waren.

Fig. 6. - Migration des spermatozoïdes (en centaines) dans des spermathèques après ponction et aspiration du liquide.

\section{5. - Verhalten der Spermatozoen in den Geschlechtsorganen der Königin}

Es wurden 23 Königinnen instrumentell besamt und 6 Stunden später fixiert. Vom gesamten inneren Geschlechtstrakt wurden histologische Schnittpräparate angefertigt, bei deren Untersuchung sich folgendes Bild ergab.

Von den gefüllten Ovidukten reichte das Sperma über die Vagina bis zur Bursa copulatrix. Deutlich ließ sich im medianen Ovidukt ein " orientierendes Verhalten 》 (F. RutTNER, 1956) der Spermatozoen erkennen, das sich daran zeigte, daß sie mit den Köpfen zur Wand gerichtet waren. Die Mündung des Samenblasenganges umsäumte ein geordnetes Bündel parallel gelagerter Spermatozoen, das bis in den Gang hineinragte (Abb. 7). Die Hauptmasse der Spermatozoen lag aber als ungeordneter Knäuel in den Ovidukten. Sie wird offenbar durch die Vagina ausgepreßt.

Das Lumen des Samenblasenganges wird durch eine Chitinlage von unterschiedlicher Dicke ausgekleidet. Am stärksten $(2-3 \mu \mathrm{m})$ ist sie in der Kontraktionszone ausgebildet. Der Durchmesser des Ganges beträgt an der Mündung in den mittleren Eileiter 20-25 $\mu \mathrm{m}$, im Bereich der Samenpumpe verengt er sich auf 8-10 $\mu \mathrm{m}$, um sich im Bereich der Einmündung der beiden Drüsenäste bis auf 25-35 $\mu \mathrm{m}$ Durchmesser zu erweitern. Diese Messungen wurden an fixiertem Material durchgeführt.

Im Samenblasengang liegen die Spermatozoen dicht gepackt in wellenförmiger Anordnung. In der trichterförmigen Erweiterung in Höhe der Ein- 
mündung der Spermathekaldrüse ist der Hauptstrang der Spermatozoen zur Spermatheka gerichtet. Eine Untersuchung der etwa 6-8 $\mu \mathrm{m}$ weiten Hauptgänge der Drüsen haben aber ergeben, daß auch diese dicht mit Spermien angefüllt waren. Einzelne Spermatozoen waren sogar bis in die Zellhalskanäle vorgedrungen (Abb. 8).

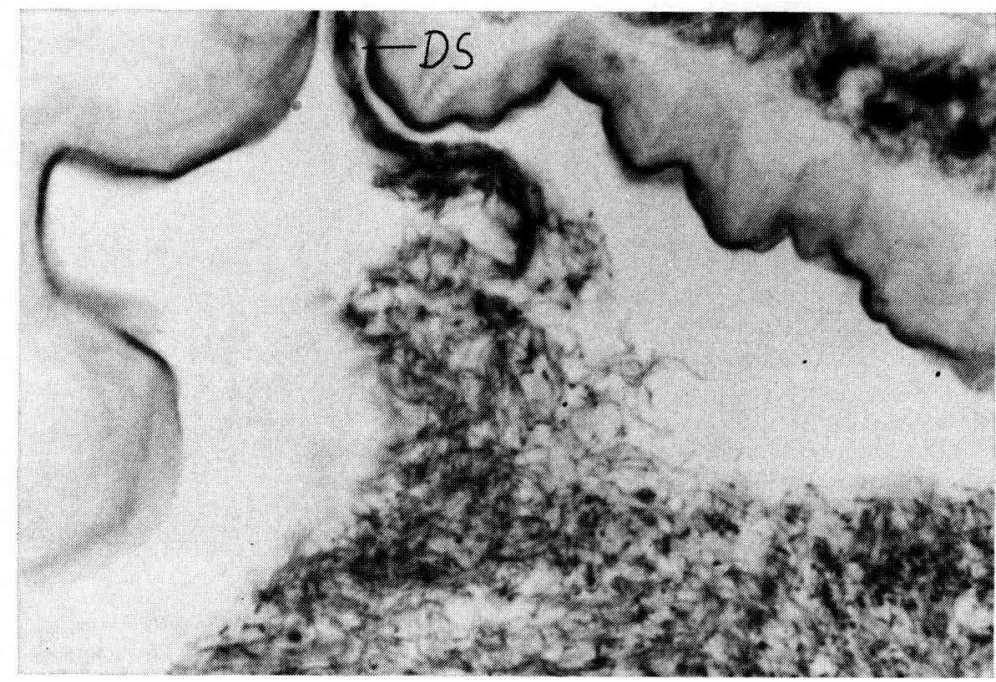

Aвв. 7. - Mündung des Ductus spermaticus in die Vagina.

Der Grossteil der Spermien befindet sich in einer ungeordneten Masse (unten), die später durch die pressenden Bewegungen der Königin ausgeschieden wird. Nur ein Teil der Spermien bewegt sich mit parallel angeordneten Geisseln aktiv in Richtung Ductus (oben). DS Ductus spermaticus. Vergr. ca 280 fach.

FIG. 7. - Orifice du ductus spermaticus dans le vagin.

La majeure partie des spermatozoïdes se trouve en une masse désordonnée (en bas) qui est éliminée ensuite par les mouvements de compression de la reine. Seule une partie des spermatozoïdes, tous rangés parallèlement, se déplace activement en direction du ductus (en haut), DS : ductus spermaticus: Grossissement : environ 280

\section{DISKUSSION}

Der von Bresslau (1905) aufgrund histologischer Schnittpräparate geprägte Begriff der « Spermathekalpumpe » führte zur Vermutung aktiver Muskelkontraktionen, welche aber bisher noch nicht eindeutig nachgewiesen werden konnten. Durch die in dieser Arbeit beschriebenen reproduzierbaren Kontraktionen ist für zukünftige Untersuchungen jedoch eine Möglichkeit aufgezeigt, weitgehende Informationen über deren Mechanismus zu erhalten.

Das zu geringe Bildauflösungsvermögen der zur Verfügung stehenden Fernsehkamera gestattete es leider nicht, exakt zwischen einer peristaltischen Bewegung und einer einfachen Kontraktionsphase zu unterscheiden. Es 
reichte aber aus, um die bisherige hypothetische Annahme der Unterstützung des Spermatozoentransfers in die Spermatheka durch die Spermathekalpumpe zu bestätigen.

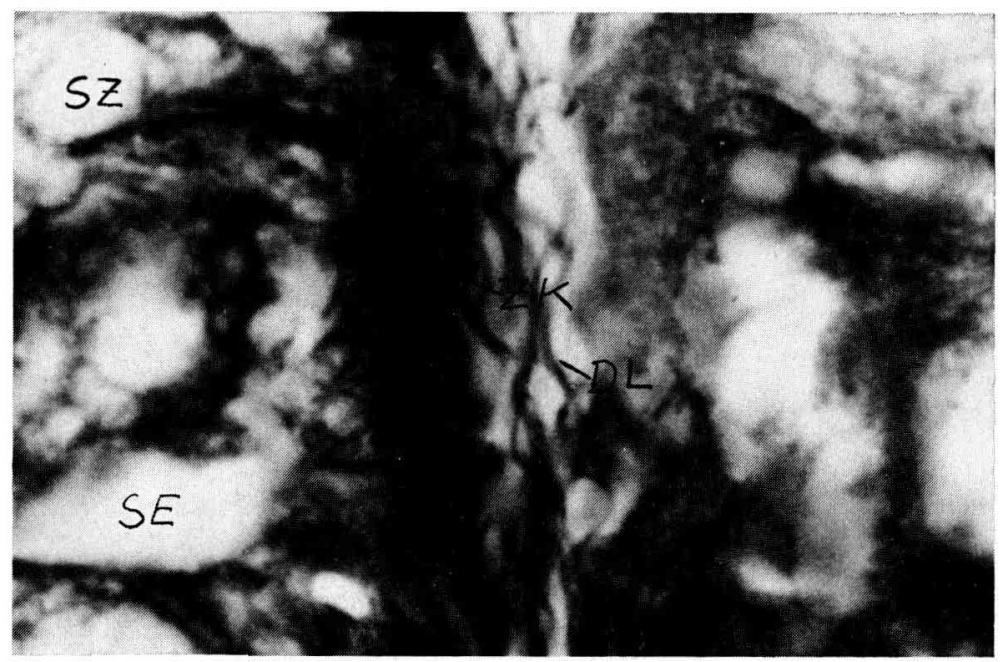

Авв. 8. - Längsschnit, durch einen Ast der Spermathekaldrüse

SE Sekret; SZ Drüsenzelle; ZK Zellhalskanal mit Spermatozoen; DL Lumen des Drïsenganges mit eingewanderten Spermatozoen. Vergr. ca 2700 fach.

Fig. 8. - Coupe longitudinale dans une branche de la glande de la spermathèque.

$\mathrm{SE}$ : sécrétion; $\mathrm{SZ}$ : cellule sécrétrice; $\mathrm{ZK}$ : canal du col de la cellule avec des spermatozoïdes; $\mathrm{DL}$ : lumière du conduit glandulaire avec des spermatozoïdes qui ont immigré.

Grossissement : environ 2700

Der passive Transport lebloser runder Partikel in die Spermatheka darf als wesentliches Argument dafür gewertet werden, daß die Kontraktionen der Muskulatur des Ductus spermaticus die Einwanderung der Spermatozoen fördern. Ein Transport inaktiver und deshalb ungeordneter Spermatozoen ist hingegen nicht möglich.

Ein zweiter wesentlicher Befund zur Deutung des Spermatozoentransfers in die Spermatheka liegt in der Beobachtung einer gerichteten Lokomotion in den Kapillargängen unterhalb eines Durchmessers von $50 \mu \mathrm{m}$. Die unter einem Öltropfen ermittelte optimale Wanderungsgeschwindigkeit bei einem Gangdurchmesser unter $35 \mu \mathrm{m}$ liegt damit im Bereich des Durchmessers des Ductus spermaticus.

Die Punktion der Spermatheka verhinderte nicht das Einwandern der Spermatozoen. Die Annahme einer möglichen Rheotaxis der Spermien durch entgegenströmende Flüssigkeit ist damit widerlegt. Offen bleibt hingegen die 
Möglichkeit der Chemotaxis durch das Drüsensekret bzw. die Spermathekalflüssigkeit. Auch die Entfernung der Drüsen (Ruttner und Koeniger 1971) konnte dieses Problem nicht lösen. Denn es war nicht zu unterscheiden, ob das Drüsensekret über eine einfache Aktivierung oder über eine echte Chemotaxis wirkt.

Nach Absaugen der Spermathekalflüssigkeit wandern nur in den ersten Stunden nach erfolgter Besamung Spermatozoen ein, später jedoch nicht mehr. Es ist anzunehmen, daß die anfängliche Einwanderung durch die im Ductus verbliebene Flüssigkeit ermöglicht wurde. Sobald diese durch die aus der Spermatheka nachströmende Hämolymphe ersetzt war, kam die Spermienmotilität zum Erliegen.

Einen entscheidenden Hinweis auf eine gerichtete Lokomotion und die Art der Motilität offenbarten histologische Schnittpräparate. Es wird deutlich, daß der Situs der Spermatozoen in den Geschlechtsgängen der Königin wenige Stunden nach der Besamung mit den experimentellen Befunden in Einklang steht :

Im medianen Ovidukt orientieren sich die an der Oberfläche liegenden Spermatozoen durch Eigenbewegung zur Wand. In der trichterförmigen Mündung des Samenblasenganges entsteht auf diese Weise ein zur Samenblase hin orientierter Spermienstrang. Die Spermamasse im Zentrum des Eileiters hingegen bleibt ungeordnet und wird in diesem Zustand durch die Vagina nach außen befördert. Bei ihrer Wanderung in die Spermatheka dringt ein Teil der Spermatozoen auch in die Gänge der Samenblasendrüse ein.

Aufgrund dieser Ergebnisse kann das eingangs dargestellte Blockdiagramm wesentlich vereinfacht werden (Abb. 9). Für den Transport der Spermatozoen von den Ovidukten in die Samenblase ergibt sich nun folgender Weg :

1. Durch Kontraktionen der Abdominalmuskulatur der Königin (B) werden die Spermamassen in Richtung der Mündung des Samenblasenganges gepreßt.

2. Unterdessen kommt es durch die “ Wandreaktionen » der Spermatozoen (d.h. ihr Verhalten, so lange schlängelnde Suchbewegungen auszuführen, bis sie mit ihrem Kopf an eine Oberfläche -. die Wand des Organs, Luftblasen, feste Partikel - stoßen) zu einer wenigstens vorübergehenden Anheftung und damit zu einer polaren Orientierung der Spermatozoen (C). In einen engen Gang mit trichterförmiger Öffnung gelangen deshalb zwangsläufig nur polar orientierte Spermatozoen. Damit wird verständlich, warum nur ein kleiner Teil der übertragenen Spermatozoen die Spermatheka erreicht.

3. Die in die Mündung des Ductus spermaticus gelangten Spermien bewegen sich mit beachtlicher Geschwindigkeit in Richtung zur Spermatheka $(\mathrm{F})$. Ihre Eigenbeweglichkeit hängt ab 
a) vom Chemismus des umgebenden Mediums und

b) von dem zur Verfügung stehenden Raum, wie eindeuti durch ihre Bewegungen unter dem Öltropfen belegt wurde.

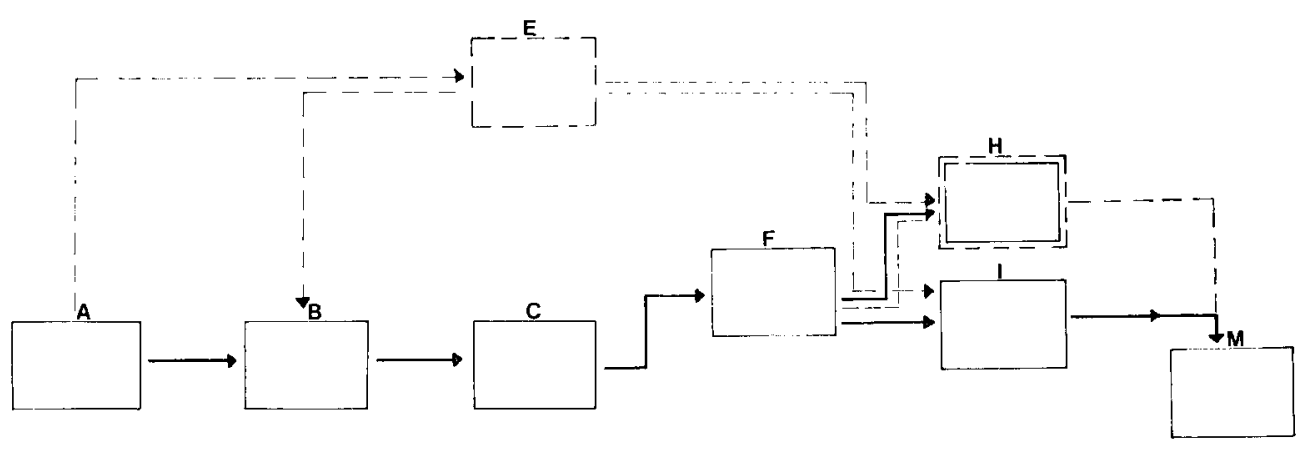

Авв .9. - Diagramm zum Spermientransfer in die Spermatheka unter Berïcksichtigung der hier vorgelegten Ergebnisse (s. Abb. 1). Erläuterungen siehe Text.

FIG. 9. - Diagramme du transfert des spermatozoïdes dans la spermathèque d'après les résultats exposés ici (voir Fig. 1).

Explications dans le résumé

4. Die Erweiterung des Raumes in der Spermatheka (M) hat ein Einrollen und damit eine Behinderung der nachfolgenden Spermien zur Folge. Die Kontraktionen der Spermathekalpumpe (I) sorgen aber für einen kräftigen Schub des Spermienstranges. Die Bedeutung dieser passiven Transporthilfe ist vermutlich in der letzten Phase der Spermathekalfüllung besonders groß, wenn es gilt, eine möglichst dichte Packung zu erzielen.

5. Die Frage nach der spezifischen Wirkung des Drüsensekretes (H) kann noch nicht eindeutig beantwortet werden. Ihr Einfluß auf die Aktivität und Lebensdauer der Spermatozoen geht aus früheren Untersuchugen hervor.

$$
\text { Eingegangen im September } 1976
$$

Reçu pour publication en septembre 1976

\section{RESUMÉ}

Lors de l'insémination de la reine d'abeille, le sperme est tout d'abord emmagasiné dans les oviductes latéraux. De là une partie des spermatozoïdes parvient à la spermathèque dans Ies 24 heures, tandis que la majeure partie d'entre eux est expulsée vers l'extérieur par le vagin. Il est bien connu que, lors de ce transfert, les mouvements passifs aussi bien qu'actifs des sper- 
matozoïdes jouent un rôle (RUTTNer et KoEniger, 1971), mais il reste encore à élucider l'importance des facteurs pris isolément. Ce problème a été réexaminé à l'aide d'autres méthodes.

Sur des préparations fraîches on a pu déclencher des contractions des muscles du ductus spermaticus en les arrosant d'une solution de Ringer tiède. Une contraction annulaire particulièrement nette se forme dans la région où le canal du ductus est particulièrement étroit (8 à $10 \mu \mathrm{m}$ ) et pourvu d'une épaisse couche de chitine. A l'aide de ces contractions musculaires on a pu également envoyer dans la spermathèque des corps inertes.

On a injecté dans les oviductes $6 \mu \mathrm{l}$ d'une suspension de billes de polyéthylène ( $\varnothing 7 \mu \mathrm{m}$, nombre 4,5 millions) et trouvé en moyenne 1140 billes dans la spermathèque.

La migration des spermatozoïdes a été étudiée dans des canaux étroits, emplis d'un liquide et extraits à l'aide d'une canule sous une goutte d'huile de paraffine (Fig. 3). Dans les canaux de moins de 35 um de diamètre (Fig. 4) la vitesse de locomotion est la plus élevée. La migration est plus rapide dans l'hémolymphe que dans le liquide spermathécal, mais les spermatozoïdes sont inactifs un peu plus tard.

Pour étudier une éventuelle stimulation rhéotactique sur les spermatozoïdes (RUTrNer et Koeniger, 1971), on a effectué avant l'insémination une ponction dans la spermathèque aux glandes intactes. La migration des spermatozoïdes a malgré tout eu lieu dans des proportions normales (Fig. 5).

Si par contre on prélève simultanément le liquide spermathécal en l'aspirant - l'espace vide se remplit alors automatiquement d'hémolymphe - le remplissage de la spermathèque se trouve alors interrompu. Ceci prouve que la migration des spermatozoìdes dépend de la nature du liquide de la spermathèque. Lors de la migration, les spermatozoïdes se trouvent dans un état hautement actif; il s'ensuit qu'ils migrent même dans les canaux des glandes (Fig. 8).

Le comportement des spermatozoïdes dans l'oviducte et le canal de la spermathèque a été étudiẻ à l'aide de coupes histologiques (Fig. 7).

On a cherché à représenter l'ensemble complexe des facteurs qui entrent en jeu dans le transfert des spermatozoïdes au moyen de diagrammes, l'un sans tenir compte des nouveaux résultats, l'autre en en tenant compte (Fig. 1 et 9). Situation de départ (Fig. 1) : Lors de l'insémination la majeure partie du sperme parvient dans les oviductes (A) et en dilate les parois. Cela déclenche probablement une stimulation des récepteurs, qui provoque une excitation du système nerveux central (E). Sur les trajets nerveux il pourrait se produire un déversement de sécrétion glandulaire $(\mathrm{H})$ ainsi qu'une activation de la " pompe spermathécale " qui pourrait consister en de simples contractions musculaires (I) ou en un véritable effet de pompe (aspiration du sperme, K). A la suite de l'insémination on peut observer de fortes contractions de l'abdomen (B), qui provoquent un transport du sperme en direction caudale. En raison du faible diamètre du ductus spermaticus seuls les spermatozoïdes disposés parallèlement et orientés polairement peuvent le traverser; les spermatozoïdes inactifs restent en un rouleau désordonné (D) et sont expulsés par le vagin. Les spermatozoïdes actifs s'orientent la tête vers la paroi et peuvent atteindre la spermathèque (M), soit par migration active (F), soit par transport passif (G). Le transport pourrait être facilité par l'effet d'aspiration de la pompe spermathécale (K). La migration active pourrait être favorisée par la rhéotaxie (N) de sens opposé à un courant provenant de la spermathèque $(\mathrm{L})$, par la simple activation due à la sécrétion glandulaire $(\mathrm{H})$ ou par les contractions de la pompe (I).

En tenant compte des résultats de ce travail, le diagramme peut être substantiellement simplifié (Fig. 9) : les facteurs les plus importants demeurent les contractions des muscles abdominaux de la reine (B), l'orientation polaire (C) et la migration active (F) des spermatozoïdes, favorisée par la sécrétion glandulaire activante $(H)$ et par les contractions des muscles du ductus spermaticus $(\mathrm{I})$. Les phénomènes au niveau du système nerveux central de la reine $(\mathrm{E})$ n'ont pas été étudiés et restent donc hypothétiques. 


\section{LITERATUR}

Bresslau E., 1905, Der Samenblasengang der Bienenkönigin. Zool. Anz. 29, 299-325.

IfaNTidis M.D., 1972, Einfluss von Aufzuchtbedingungen und Alter auf die Ausbildung von Spermien der Honigbiene. Bonn : Inaugural-Dissertation,

Koeniger G., 1970, Bedeutung der Tracheenhülle und der Anhangdrüse der Spermatheka für die Befruchtungsfähigkeit der Spermatozoen in der Bienenkönigin. Apidologie 1, 55-71.

Rothschild Lord, 1955, The spermatozoa of the honeybee. Trans. R. Soc. London 107, 289294.

Ruttner F., 1956, Zur Frage der Spermaübertragung bei der Bienenkönigin. Ins. soc. 3, 351 359.

Ruttner F. (Herausgeber), 1975, Die instrumentelle Besamung der Bienenkönigin. Apimondia Verlag, Bukarest.

Ruttner F., Koeniger G., 1971, Die Füllung der Spermatheka der Bienenkönigin. Aktive Wanderung oder passiver Transport der Spermatozoen? Z. vergleich. Physiologie 72, 411422. 